\title{
AN EXTENDED CONTACT MODEL OF THE ANGULAR BEARING
}

\author{
JAN KOSMOL \\ Silesian University of Technology, Gliwice, Poland \\ e-mail: jkosmol@polsl.pl
}

\begin{abstract}
The article presents a new contact model of an angular bearing, called the extended model. The model takes account of the effects of centrifugal load caused by thr rotating ring of the bearing and the issue of elasticity. Present models, encountered in literature and referred to in the article as classical, consider the centrifugal force caused by the rotating ball only. Results of analytical research on the extended model and of FEM simulation show explicitly that the contact angles of bearings, and thus contact loads, differ very much from those values obtained in the classical model. These differences are disadvantageous for designing bearing assemblies, since contact loads are greater than those obtained in the classical model. This means, among others, that the present structures with angular contact bearings are in reality subject to greater loads than the constructor has envisaged. The motto of the article is to design analytical calculation models in such a form which would enable the constructor to estimate contact loads, just using a standard scientific calculator or MS Excel type applications; so that there would be no need for solving complex models using numerical methods.
\end{abstract}

Keywords: contact model, angular bearing, FEM

\section{Introduction}

One of the major constructional issues of HSC machine tools is the heat generated in rolling-contact bearings, e.g. in bearing seatings of high-speed spindles. The designer of the bearing seating should have a possibility of estimating the amount of heat generated in the bearing, so that he would be able to foresee, if needed, the necessity of applying a cooling system. In order to estimate the amount of heat generated in the bearing, there is the need for, among others, information on motion resistance existing in the bearing. Motion resistance in the bearing, measured on the driving shaft, is determined according to the so-called formula (Palmgren, 1951), i.e.

$$
M_{f}=M_{1}+M_{v}
$$

where $M_{f}$ is the total bearing friction torque, $M_{1}$ - friction torque due to external load, $M_{v}-$ friction torque due to lubrication.

Motion resistance due to the lubricant or oil $M_{v}$ can be determined using common mathematical formulae (Harris and Kotzalas, 2013). At the some time, motion resistance due to rolling friction in the bearing $M_{1}$, according to Palmgren (1951) formula, is represented as follows

$$
M_{1}=z\left(\frac{F_{s}}{C_{s}}\right)^{y}\left(0.9 F_{a} \cot \alpha-0.1 F_{r}\right) d_{m}
$$

where: $F_{a}, F_{r}$ are axial and radial loads, $F_{s}$ - bearing static equivalent load, $C_{s}$ - bearing basic static load rating, $\alpha$ - contact angle, $z, y$ - coefficients dependent on the bearing structure and the lubrication method. 
It presents results approximating reality for relatively low rotational speeds. As rotational speeds increase, the difference between the friction torque calculated according to formula (1.1), and the real motion friction torque, increases. Hence, the amount of heat generated in the bearing, calculated according to formula (1.1), is lower than in the real system.

Technical lliterature draws attention to numerous models which take account the influence of rotational speed, applied bearing load and preload on contact angles in angular bearings and on contact loads $Q$ taking place between the ball or shaft and the raceways of bearing rings (Alfares and Elshrakawy, 2003; Antoine et al., 2005; Chen and Hwang, 2006; Jiang and Mao, 2010). Friction torque $M_{1}$ as per Musiał and Styp-Rekowski (1999), Styp-Rekowski (1999) is a function of contact loads $Q$ and rolling friction coefficient $f$ between the rolling element and bearing raceways. Hence, familiarity of contact loads $Q$ and the assumption of rolling friction coefficient $f$ enabled determination of the friction torque $M_{1}$.

Harris and Kotzalas (2013) presented a model which takes into account the influence of preload on the bearings contact angle $\alpha$ and on the contact load $Q$. This model assumes that contact of the bearing ball with raceway falls under Hertz theory, whereas the mathematical formula connecting the contact load with contact deformation has been assumed under the form of Jones model (Haris and Kotzalas, 2013)

$$
Q=K \delta^{n}
$$

here: $K$ is rhe contact stiffness, $\delta$ - contact deformation, $n$ - constant value (for ball bearings $n=1,5$ ). Contact stiffness coefficient $K$ is a function of ball curvature and bearing raceways.

Several authors, among others, Alfares and Elsharkawy (2013), Altintas and Cao (2005), Chen and Hwang (2006), Cao and Altintas (2007), Jiang and Mao (2010), Jędrzejewski and Kwaśny (2010), Abele et al. (2010), and Noel et al. (2013) have taken account the effects in contact zones, which generates centrifugal load from rotating raceways, balls or shafts. One of these is the difference in the bearings contact angles on inner and outer raceways and their dependence on rotational speed. These models take into account simultaneous application of the preload force of the angular bearing and the centrifugal load. A notable progress in the development of modelling contact effects in bearings was the work of Antoine et al. (2005). Considering the case of the bearings preload with the use of a spring, they presented an analytical relationship between the bearings contact angles, present on both raceways, the inner and outer one. This dependence has a substantially facilitated analytical solution in calculating contact angles.

Few are publications on calculation of the total friction torque. The exceptions are some articles (Musiał and Styp-Rekowski, 1999; Styp-Rekowski, 1999). Analytical formula has been presented, which enables calculation of the friction torque on the driving shaft, basing on the familiarity of contact loads and on the rolling friction coefficients. A common characteristic of all encountered in literature contact models of angular bearings is the assumption of non-deformity of all elements of the bearings except for the ball or shaft. Only contact deformations of the ball or shaft are considered in calculation of the contact angles or contact loads (Fig. 1a)

A second common characteristic relates to the loads. The loads resulting from rotating rolling elements are considered in analysis of contact effects only. At the same time, there have been completely disregarded the loads resulting from the rotating inner ring of the bearing and resulting from deformation of the ring due to the centrifugal force.

\section{Classical contact model of the angular bearing}

In order to compare the used till now contact models of angular rolling bearings with the recommended, new extended model, there shall be presented both models on the example of an angular bearing subject to the preload $F_{a}$ and centrifugal load $F_{c}$, arising from rotating balls. 

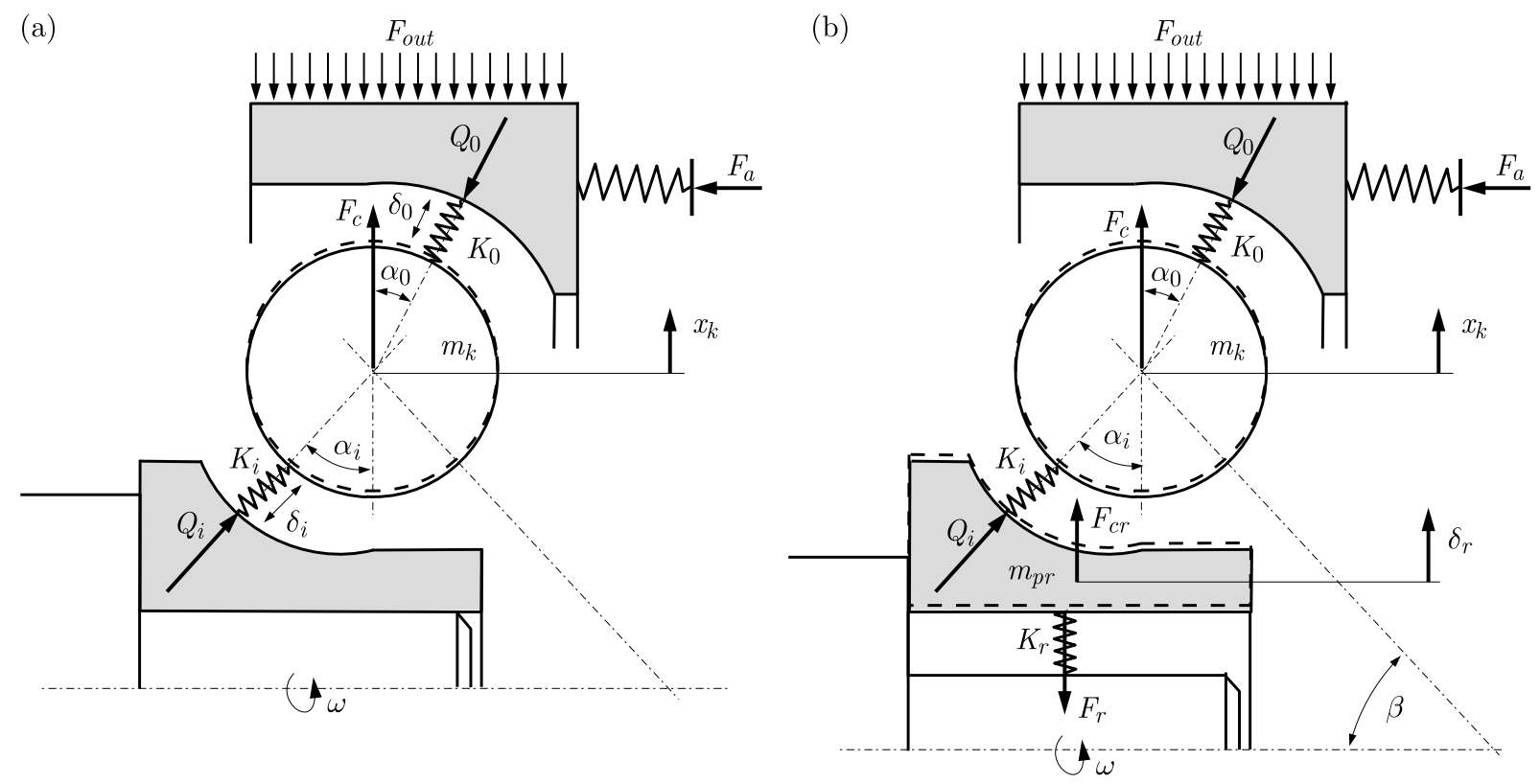

Fig. 1. Contact models of angular bearings: (a) classical, (b) extended

The procedure of solving the contact problem in angular bearings boils down to determination of the contact angles. Their familiarity enables determination of the other parameters, e.g. contact loads. The procedure can be split down into at least three steps:

- identification of the position of centres of curvatures of the bearing inner and outer raceways and of the ball centre with the bearing being subject to load and not being subject to loads,

- generation of conditions of equilibrium of loads acting on the bearings elements,

- generation of the geometrical condition, which shall enable determination of one of the contact angles.

Figure 2 presents the state of the angular bearing subject only to the preload $F_{a}$ (Fig. 2a) as well as preload $F_{a}$ and the centrifugal load $F_{c}$ from the rotating ball (Fig. 2b).

For configuration depicted in Fig. 2a, the position of centres of raceway curvatures and of the centre of the ball in a state not subject to the load is indicated as $i, o$ and $O$. Geometric constructional characteristic of such a state is the distance between points $i$ and $o$, marked as $A$. It is a constructional characteristic of each bearing. Equally important constructional characteristics of bearing are the radii of curvature of the bearing raceways $r_{i}$ and $r_{o}$, as well as the contact angle $\alpha$.

After the bearing is subject to the preload $F_{a}^{*}$ (in this example applied to the inner ring) displacement of the inner ring takes place. As a result the centre of curvature of the inner raceway is displaced into the position $i^{*}$. This means that the distance between the points $i^{*}$ and $o$ changes and assumes the value $A_{p}$ and the contact angle assumes the value $\alpha_{p}$.

In the load configuration depicted in Fig. $2 \mathrm{~b}$ (preload and centrifugal load of the rotating ball), the centres of curvatures of both raceways $i^{*}$ and $o$ are not subject to change, however the ball centre gets displaced to point $O_{1}$. At this stage of resolving the contact problem, there is also determined the distance between the centres of curvatures, in this case it is $A_{p}$.

The second step of the procedure resolving the contact problem defines the conditions of equilibrium of the loads acting on the bearing elements. For instance, for configuration depicted 
(a)

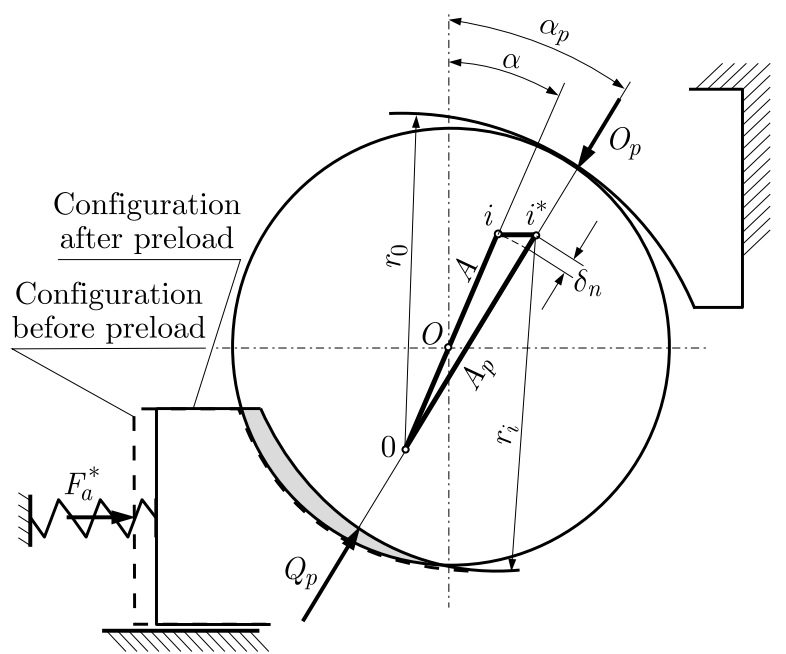

(b)

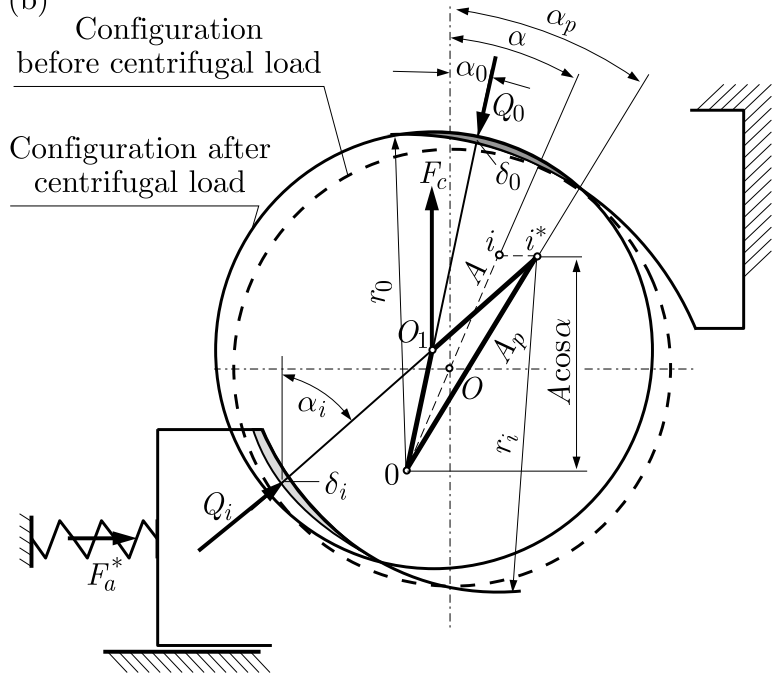

Fig. 2. Configurations of the angular bearing subject to: (a) preload $F_{a}^{*}$ and (b) preload $F_{a}^{*}$ and centrifugal load $F_{c}$ from rotating bearing balls: $\delta_{i}, \delta_{o}$ - contact deformations, $Q_{i}, Q_{o}, Q_{p}-$ contact loads, $\alpha, \alpha_{i}, \alpha_{o}, \alpha_{p}$ - contact angles, $r_{i}, r_{o}$ - raceway curvature radii, $i$ - index relating to the inner raceway, $o$ - index relating to the outer raceway, $p$ - index relating to the state of preload $F_{a}^{*}$

in Fig. 2b (simultaneous preload and centrifugal load from the rotating ball), from the equations of equilibrium of loads acting on the ball, it follows that

$$
Q_{i} \sin \alpha_{i}=Q_{o} \sin \alpha_{o} \quad F_{c}=Q_{i} \cos \alpha_{i}-Q_{o} \cos \alpha_{o}
$$

From the conditions of equilibrium of loads acting on the inner and outer ring, it can be seen that

$$
F_{a}^{*}=Q_{i} \sin \alpha_{i} \quad F_{a}^{*}=Q_{o} \sin \alpha_{o}
$$

If relationships (2.2) are inserted into the second equation of (2.1), we obtain a very interesting relationship, coupling together both contact angles as

$$
\frac{1}{\tan \alpha_{o}}-\frac{1}{\tan \alpha_{i}}=\frac{F_{c}}{F_{a}^{*}}
$$

Relationship (2.3), published by Antoine et al. (2005), is very significant for resolving the contact problem of angular bearings as it reduces the number of unknowns by 1 .

The third step of the procedure resolving the contact problem, defines the geometrical condition, which shall enable determination of the single contact angle. The geometrical condition includes, among others, contact deformations $\delta_{i}$ and $\delta_{o}$ as well as the distance connecting the centres of curvatures $i$ or $i^{*}$ and $o$, i.e. $A$ or $A_{p}$.

Formulation of the geometrical condition, for example, for configuration depicted in Fig. $2 \mathrm{~b}$ is somewhat more complicated. For the triangle $o i^{*} O_{1}$, it can be stated as follows

$$
i^{*} O_{1} \cos \alpha_{i}+O_{1} o \cos \alpha_{o}=A \cos \alpha
$$

Since $i^{*} O_{1}$ and $O_{1} O$ can be expressed as

$$
i^{*} O_{1}=r_{i}+\delta_{i}-\frac{1}{2} D \quad O_{1} O=r_{o}+\alpha_{o}-\frac{1}{2} D
$$

then, by inserting (2.5) into (2.4) we obtain the sought geometrical condition

$$
\left(r_{i}+\delta_{i}-\frac{1}{2} D\right) \cos \alpha_{i}+\left(r_{o}+\delta_{o}-\frac{1}{2} D\right) \cos \alpha_{o}=A \cos \alpha
$$


If relationship (2.6) considers equations (1.3), then it should assume the following form (Kosmol, 2016)

$$
\left(r_{i}+\sqrt[n]{\frac{F_{a}^{*}}{\sin \alpha_{i} K_{i}}}-\frac{1}{2} D\right) \cos \alpha_{i}+\left(r_{o}+\sqrt[n]{\frac{F_{a}^{*}}{\sin \alpha_{o} K_{o}}}-\frac{1}{2} D\right) \cos \alpha_{o}=A \cos \alpha
$$

Equations (2.7) and (2.3) form a system of equations, the solution to which determines the contact angles $\alpha_{i}$ and $\alpha_{o}$ in function of the centrifugal load $F_{c}$ and preload $F_{a}^{*}$.

Knowing the contact angles, we can calculate contact loads by using formulae (2.1).

Figure 3 features the influence of rotational speed on the contact loads $Q_{i}$ and $Q_{o}$ for selected values of the preload determined by the presented methodology.

(a)

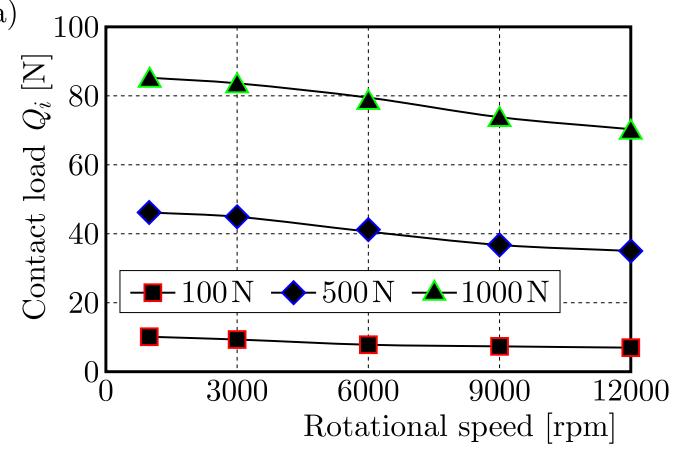

(b)

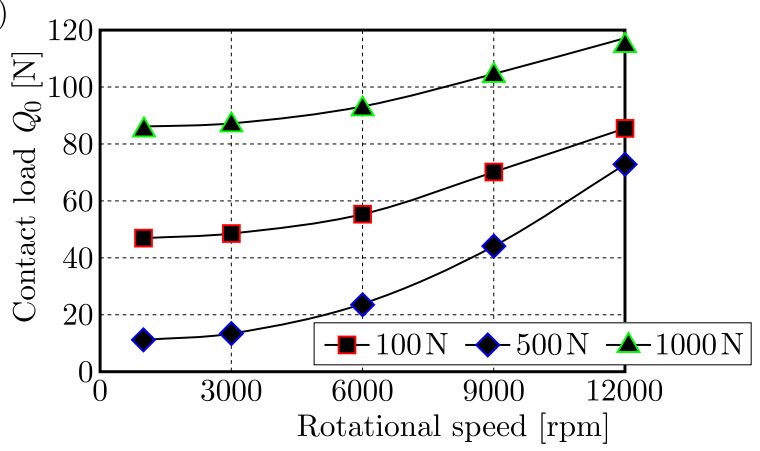

Fig. 3. Influence of rotational speed of the bearing on contact loads: (a) on the inner raceway $Q_{i}$,

(b) on the outer raceway $Q_{o}$, for selected values of the preload $F_{a}$

The simulation results in Fig. 3 confirm the known regularities that the contact load $Q_{i}$ on the inner raceway slightly decreases with an increased rotational speed, whereas on the outer raceway $Q_{o}$, it increases substantially with the increased speed.

\section{Extended contact model of angular bearings}

As already mentioned, the present contact models of angular bearings take into account just contact deformations of balls. Section 1 shows in form of an example what centrifugal load is generated by the rotating inner bearing ring and what are its radial deformations. These values are big enough to presume that they can change considerably the conditions of contact of the ball with the raceways. Hence, a concept has been conceived of a contact model of the bearing, also known as the extended model. It is depicted in Fig. $1 \mathrm{~b}$.

Figure 4 portrays the extended contact model of the bearing in convention of the classical model (Fig. 2b).

The procedure of solving such a contact problem is the same as presented in Section 2 . First of all identified are the positions of centres of curvatures of both raceways of the bearing. The starting point is the configuration corresponding to the force of preload, i.e. similar to that presented in Fig. 2a. The centres of curvatures are in points $i^{*}$ and $o$, and the section connecting both points has length $A_{p}$. The bearing contact angle is $\alpha_{p}$. The emergence of the centrifugal load $F_{c r}$ of the rotating bearing ring shall cause expansion of that ring and, in effect, the displacement in the radial direction $\delta_{r}$. In consequence, the centre of curvature of the inner raceway is displaced, which assumes position $i^{* *}$ (Fig. 4). The displacement of centre of curvature of the inner raceway from point $i^{*}$ to point $i^{* *}$ is equal to the displacement $\delta_{r}$. The section connecting the points $i^{* *}$ and $i^{*}$ has length $A_{c r}$, and the inclination angle of that section is $\alpha_{c r}$. 

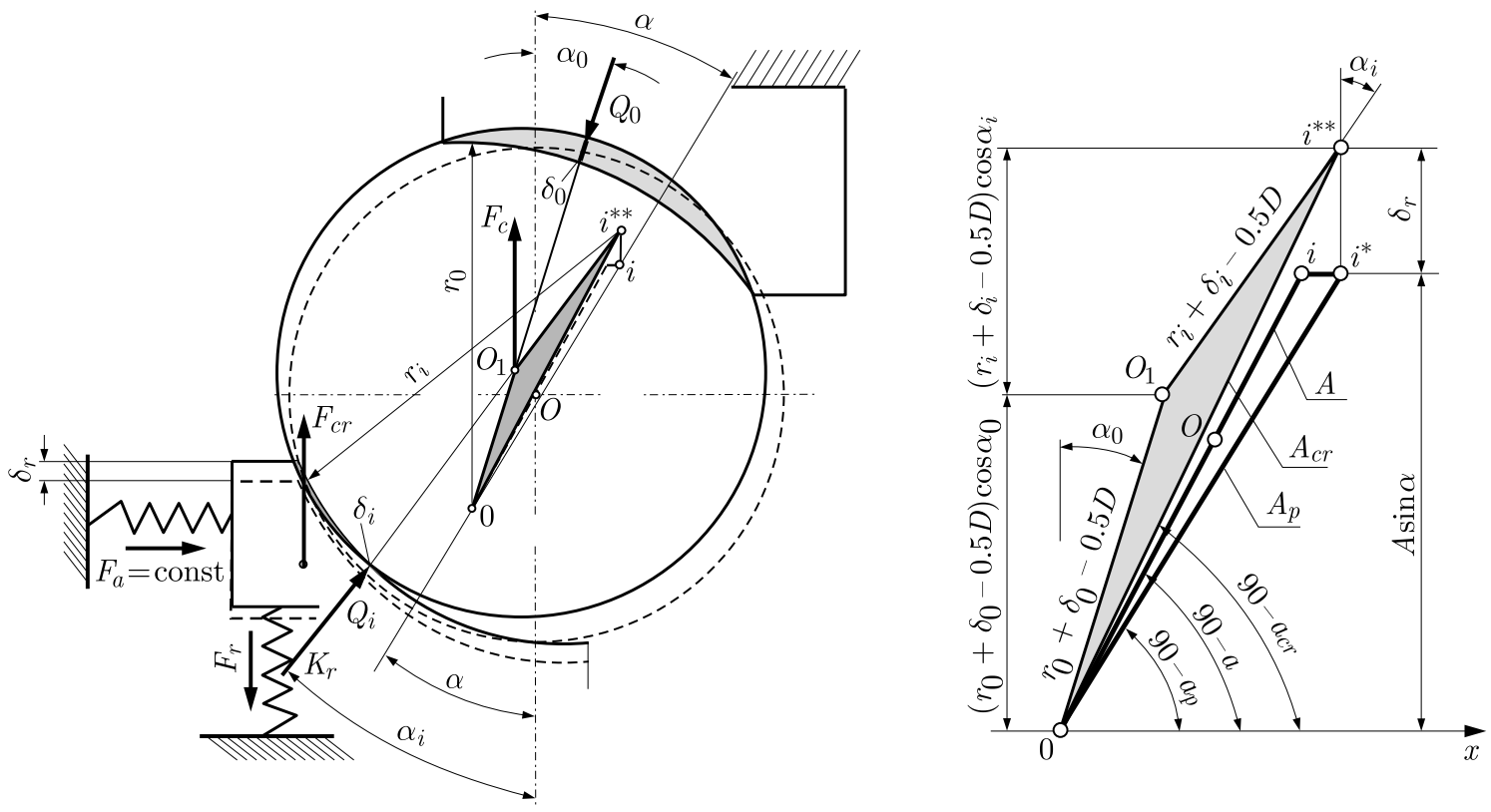

Fig. 4. Extended contact model of the bearing

In effect of action of the centrifugal load $F_{c}$ from the rotating ball, the ball centre is displaced to point $O_{1}$. As a result, there can be formed a triangle $i^{* *} i^{*} O_{1}$, which enables one to formulate the geometrical condition.

In the second step, we define conditions of equilibrium of loads acting on the bearing elements. These are the same equations as (2.1) and (2.2), and resulting relationship (2.3). A new equation, with respect to the bearings configuration, as presented in Fig. 3b, is the equation of equilibrium of loads acting on the inner ring of the bearing (Fig. 4), namely

$$
F_{c r}-F_{r}-Q_{i} \cos \alpha_{i}=0
$$

where: $F_{c r}$ is the centrifugal load from the rotating bearing ring acting on a single bearing ball, $F_{r}$ - elastic force from elastically deformed bearing ring.

By assuming a linear model of a deformable ring in the form

$$
F_{r}=K_{r} \delta_{r}
$$

where: $K_{r}$ is the stiffness coefficient of the bearing ring, $\delta_{r}$ - elastic displacement of the bearing ring, and inserting relationship (3.2) into (3.1), and finally, by considering Jones model (1.3), we obtain the equation of equilibrium of loads acting on the inner ring

$$
F_{c r}-K_{r} \delta_{r}-\frac{F_{a}^{*}}{\tan \alpha_{i}}=0
$$

from which we determine the radial displacement $\delta_{r}$, namely

$$
\delta_{r}=\frac{1}{K_{r}}\left(F_{c r}-\frac{F_{a}^{*}}{\tan \alpha_{i}}\right)
$$

Relationship (3.4) together with (2.3) shall be the basis for determination of the contact angles. Relationship (3.4) shows that as long as the following condition is met

$$
F_{c r}<\frac{F_{a}^{*}}{\tan \alpha_{i}} \quad \delta_{r}=0
$$

then $\delta_{r}$ cannot assume negative values. 
This means that as long as condition (3.5) is met, the solution of the contact problem is the same as for the classical model (see identity (2.7)). However, when this condition is not met, both solutions, i.e. for the classical model and for the extended one, shall differ.

Since the centrifugal load $F_{c r}$ from the rotating ring is a function of rotational speed of the bearing (speed of inner ring), then the threshold speed $\omega_{g r}$ can be determined, below which condition (3.5) is met, namely

$$
\omega_{g r}<\sqrt{\frac{2 F_{a}}{m_{p w} d_{s r} \tan \alpha_{i}}}
$$

where: $m_{p w}$ is mass of the inner ring, $d_{s r}$ - average diameter of the inner ring, $\omega_{g r}$ - threshold angular speed of the inner ring.

In step three of solution of the contact problem, the geometrical condition should be defined, since there are two unknowns: contact angles $\alpha_{i}$ and $\alpha_{o}$, and just single equation (2.3). The geometrical condition, which can be formulated, is very similar to condition (2.6), as it assumes the following form

$$
\left(r_{i}+\delta_{i}-\frac{1}{2} D\right) \cos \alpha_{i}+\left(r_{o}+\delta_{o}-\frac{1}{2} D\right) \cos \alpha_{o}=A_{c r} \cos \alpha_{c r}
$$

From Fig. 4, it may be inferred that there exists the following relationship between $A_{c r}$ and $A$

$$
A_{c r} \cos a_{c r}=A \cos \alpha+\delta_{r}
$$

By inserting (3.8) into (3.7) and by applying relationships (2.2), Jones model (1.3) and relationship (3.4), we get the first equation for determining the contact angles $\alpha_{i}$ and $\alpha_{o}$

$$
\left(r_{i}+\sqrt[n]{\frac{F_{a}^{*}}{\sin \alpha_{i} K_{i}}}-\frac{1}{2} D\right) \cos \alpha_{i}+\left(r_{o}+\sqrt[n]{\frac{F_{a}^{*}}{\sin \alpha_{o} K_{o}}}-\frac{1}{2} D\right) \cos \alpha_{o}-\frac{1}{K_{r}}\left(F_{c r}-\frac{F_{a}^{*}}{\tan \alpha_{i}}\right)=A \cos \alpha
$$

Equation (3.9) together with (2.3) form a system the solution of which are the contact angles $\alpha_{i}$ and $\alpha_{o}$. When solving it, one should keep in mind condition (3.5).

Solving the system of equations (3.9) and (2.3) requires application of recurrence methods.

Further on, there shall be presented results of simulation tests of the angular bearing FAG 7013B. The tests consisted in the evaluation of the influence of rotational speed of the bearing (of inner ring) on contact angles $\alpha_{i}$ and $\alpha_{o}$, and on contact loads $Q_{i}$ and $Q_{o}$ for selected values of the preload $F_{a}$. The tests have been performed for the extended contact model of the bearing.

Figure 5a presents the influence of rotational speed on contact angles of the bearing. There are presented the results of tests for the extended model and the classical one (dashed line).

By comparing the results of tests of the influence of rotational speed on contact angles for both contact models, it should be said that the consideration of centrifugal load from the rotating bearing ring $F_{c r}$ and its elasticity, significantly affects the contact angle $\alpha_{i}$ on inner raceway. The contact angle on the inner raceway decreases with an increased speed (in the classical model this angle increases), and the model differs from the other by up to over $75 \%$.

Such differences are not observed for the contact angle $\alpha_{o}$ on the outer raceway. The runs for both models are similar, i.e. the contact angle decreases with an increased speed, and the differences do not exceed $20 \%$.

Figure 5b represents the influence of speed on contact loads $Q_{i}$ and $Q_{o}$ for selected value of the preload $F_{a}$. The diagrams put together the test results for the extended and the classical model (dashed line). 
(a)

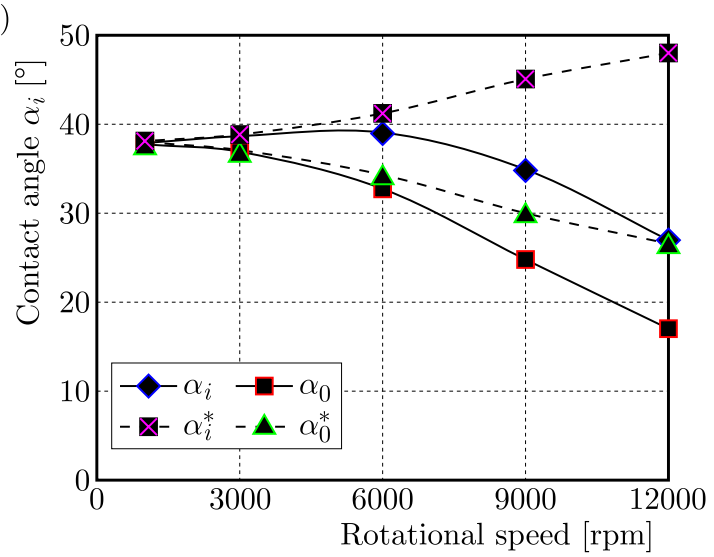

(b)

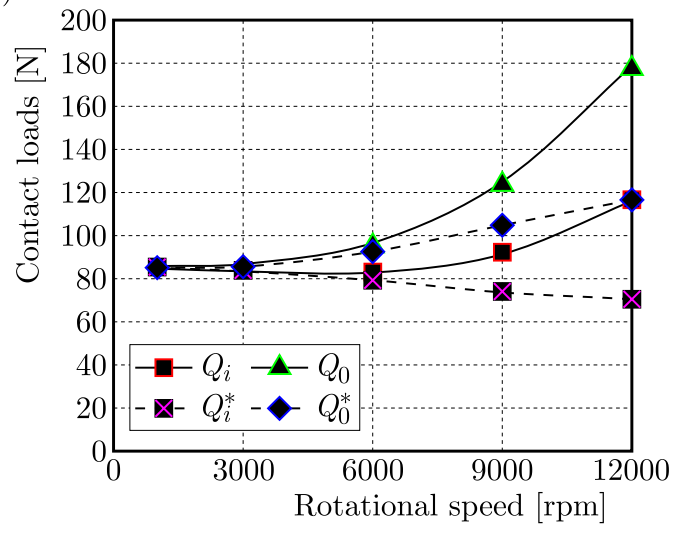

Fig. 5. Influence of rotational speed of the bearing on: (a) contact angles, (b) contact loads, for preload $1000 \mathrm{~N}$; solid line relates to the extended model, dashed line relates to the classical model

The conclusions drawn from the presented test results can have substantial practical meaning, since by generalizing, it can be said that according to the extended model, the contact loads are greater than those according to the classical model.

Qualitatively, the runs of contact loads are similar, i.e. they increase with an increased speed, however, they differ quantitatively. This refers to both, the load on the inner and outer raceways. The loads determined by both models differ by $20 \%$ to $30 \%$ to the disadvantage of the bearing, i.e. the loads determined according to the extended model are greater. This means, among others, a greater resistance to bearing motion (greater amount of heat in the bearing) and smaller durability of the latter.

On diagrams in Fig. 5, one can also observe the speed range with respect to which both models present the same results. Depending on a given preload, this relates to the speed range of 2000-2500 rotations/min, which results from inequality (3.6).

To get a full picture of the influence of centrifugal load from the rotating ring and its elasticity on contact effects in the bearing, Fig. 6a demonstrates the influence of rotational speed on radial displacements $\delta_{r}$ for the ring itself (dashed line) and for the complete bearing with three preload values.

Depending on the preload of the bearing and on its rotational speed, the difference between deformation of the inner ring alone and the displacement $\delta_{r}$ of the ring mounted in the bearing is $30 \%$.

(a)

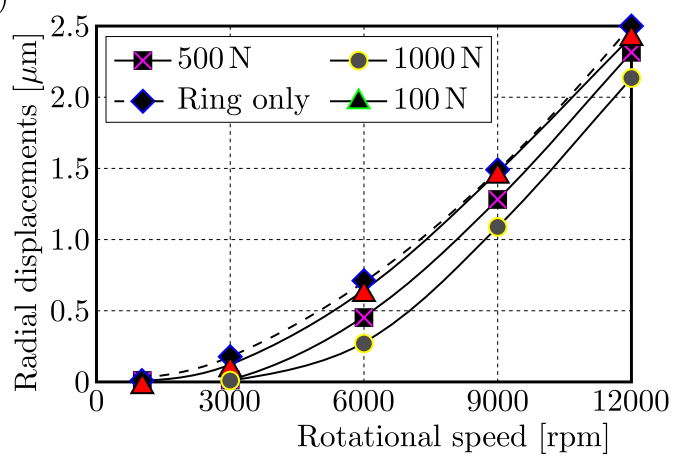

(b)

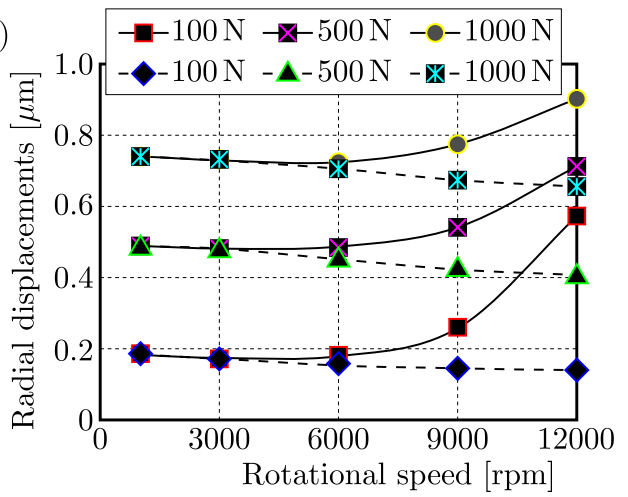

Fig. 6. Influence of rotational speed on: (a) radial displacements $\delta_{r}$ for the ring itself (dashed line) and for the complete bearing, (b) contact displacements of the inner raceway (dashed line relates to the classical model) 
However, Fig. 6b shows the influence of rotational speed on contact deformations in the zone of the inner raceway, for both contact models (dashed line relates to the classical model). Contact deformations determined for both models differ qualitatively and quantitatively, whereas these differences can reach $40 \%$ and more.

\section{Verification of the extended contact model}

Verification of the contact model of an angular rolling bearing shall be performed using two methods:

- numerical method, with the use of the finite elements method (Kosmol and Gatys, 2016),

- experimental method.

The first method can be qualified as a direct method, since it enables direct comparison of contact angles of the bearing or the effects in contact zones of the balls action with the bearings raceways (contact loads).

The second method is qualified as an indirect method, since it only facilitates comparison of the total friction torque in the bearing, which depends, among others, on contact loads.

\subsection{Numerical analysis of contact effects in bearings using the finite elements method}

The object of model tests has been the angular rolling bearing FAG B7013.

Due to the existence of numerous axes of symmetry, only a single rolling element has been modelled as well as a section of inner and outer rings.

System Ansys offers 6 ways of modelling consolidations and 5 ways of modelling contacts. Bearing rings have been deprived of the degrees of freedom corresponding to models Compression only and Friction less.

It has been decided to model contact effects with the use of the standard frictional model, which enables consideration of friction between adjacent bodies.

It is important for precision of calculations to define a calculation algorithm which predominantly determines the manner of contact detection. The Augmented Lagrange algorithm has been selected on the basis of software producer recommendations.

The preload of the bearing has been modelled in the form of thrust on the front surface of the outer ring, whereas the dynamical effects in the form of centrifugal loads acting on movable elements have been modelled using the standard Ansys mechanism.

The results of simulation are: relationships of the contact angles $\alpha_{i}$ and $\alpha_{o}$ and contact loads $Q_{i}$ and $Q_{o}$ in function of rotational speed of the bearing (of the inner ring) for a selected value of preload. Figure 7 portrays examples of the results for a preload of $1000 \mathrm{~N}$ with consideration to both the centrifugal load from the rotating ball (dashed lines) and the thrust from the bearing ring.

The contact angles determined according to the extended model are smaller than those calculated according to the conventional model. The differences measured up even to $40 \%$. But on the outer raceway, there was not so much difference observed between the angels.

A similar thing can be said about the contact loads. For the extended model, the contact loads are clearly bigger for the load on the inner and outer raceways.

By comparing the results of FEM simulations featured by Fig. 7, with the results of analytical tests, presented by Fig. 5, we observe their high qualitative similarity. Hence the results of simulation tests confirm these which have resulted from the analytical research.

Quantitative comparative evaluation of analytical and FEM methods is shown in Fig. 8. 
(a)

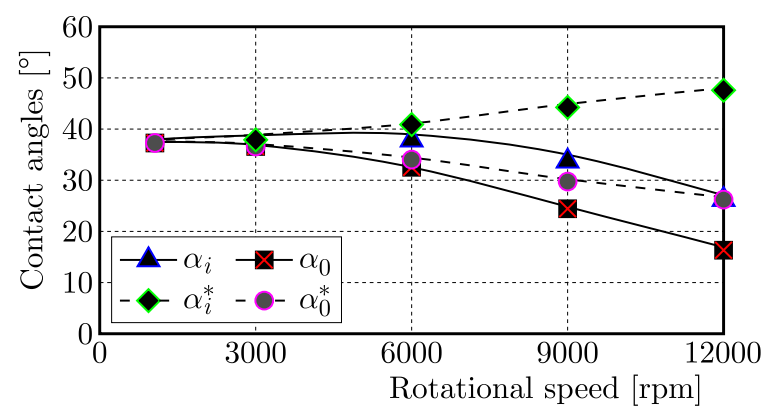

(b)

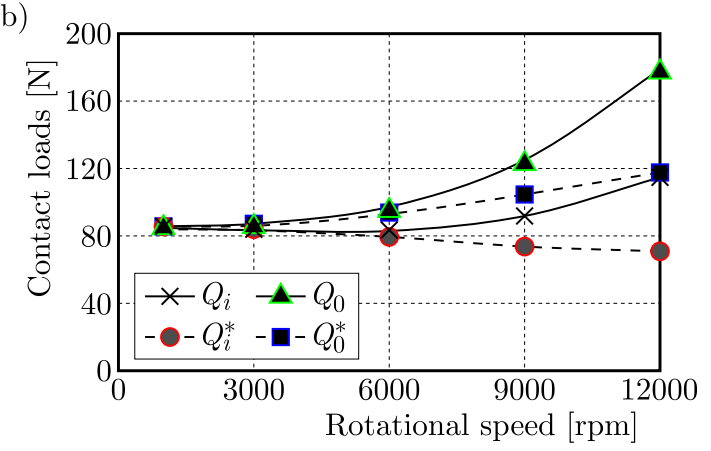

Fig. 7. Influence of rotational speed on: (a) contact angles $\alpha_{i}$ and $\alpha_{o}$, (b) contact loads $Q_{i}$ and $Q_{o}$, for preload $1000 \mathrm{~N}$; the solid line relates to a combined thrust of the rotating ball and the ring, the dashed line relates to the thrust of the rotating ball solely

(a)

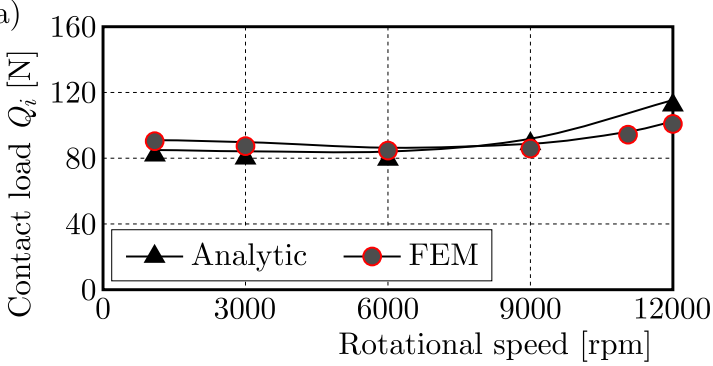

(b)

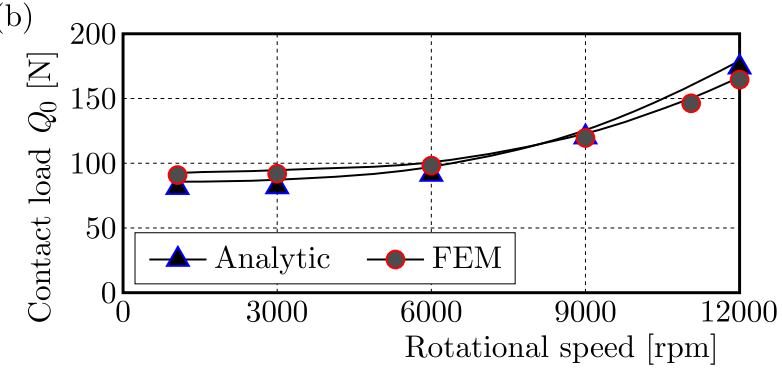

Fig. 8. Comparative evaluation of analytical and FEM methods for the assesment of the influence of rotational speed (centrifugal load from the ball and ring combined) on the contact loads: (a) $Q_{i}$, (b) $Q_{o}$, for the preload $1000 \mathrm{~N}$

A high degree of consistence is demonstrated by both calculation methods, i.e. analytical method and FEM for the contact load on the outer raceway (Fig. 8b). For the inner raceway (Fig. 8a), there are observed greater differences between both methods.

Thus, an opinion can be formulated, stating that FEM tests confirm the results of analytical tests, which constitutes a certain verification of the extended analytical model.

\subsection{Experimental verification of the extended model}

As has already been mentioned, experimental verification of the developed model can be performed through indirect methods. There are no technical possibilities for direct measurement of neither contact angles in the bearing nor contact loads. However, there exists a possibility of direct measurement of torque on the driving shaft, which is the friction torque in the bearing. Since the friction torque in the bearing is a direct function of contact loads (Musial and Styp-Rekowski, 1999; Styp-Rekowski, 1999), then, its measurement represents indirect information on the magnitude of these loads. The friction torque is also a function of coefficients of friction which should be assumed arbitrarily, guided by information provided in technical literature.

At the Department of Machine Technology of the Silesian University of Technology in Gliwice, there has been developed a research site which facilitates measurement of the torque on the driving shaft, the preload of angular bearings and the rotational speed.

A system of a spindle box, in which the spindle and two angular bearings are located, is mounted on the torque sensor made by Kistler, type 7292. The spindle drive comes from a special motor with a steplessly adjustable speed up to 18.000 rotations/min. The preload of bearings can be adjusted steplessly and measured by a sensor of the axial load manufactured by Kistler, type 9102A. 
At that site, there have been performed several tests consisting in measurement of the friction torque for certain selected values of rotational speeds and for certain values of bearing preloads (Muszyński, 2017).

As we were interested exclusively in the motion resistance of bearings depending on rolling friction of balls on bearing raceways, the bearings alone have been bereft of lubricant.

Figure 9 shows examples of the obtained results of tests of the motion resistance of two angular bearings. Figure 9a presents the influence of preload and Fig. 9b, the influence of rotational speed on the friction torque of bearings with no lubricant.

(a)

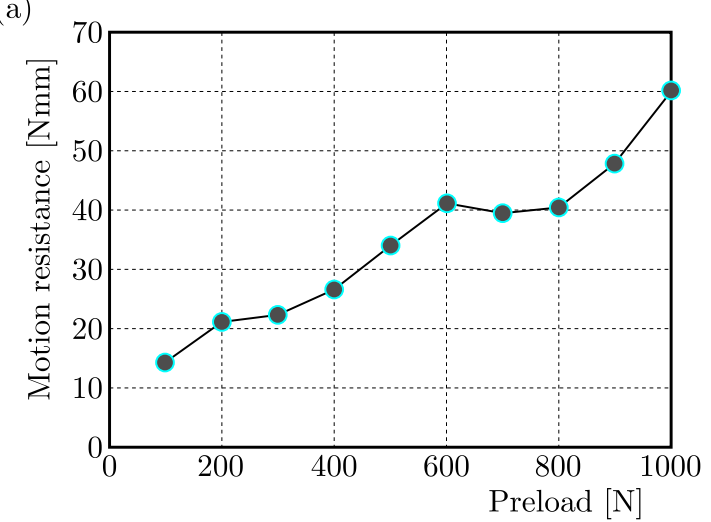

(b)

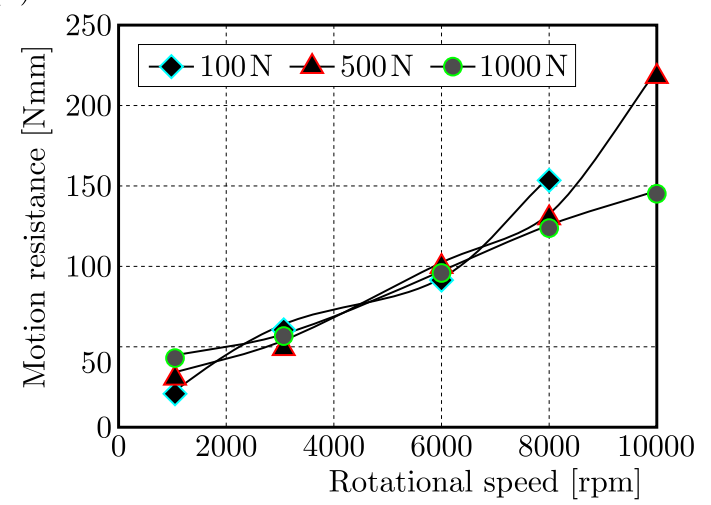

Fig. 9. Examples of measurements of motion resistance at the research site: (a) influence of preload, (b) influence of rotational speed

Thus we are speaking of the resistance resulting from rolling friction of balls which are rolling on the raceways of the bearings.

Musiał and Styp-Rekowski (1999) and Styp-Rekowski (1999) showed an analytical model of bearings motion resistance in function of contact loads, in the following form

$$
M_{1_{(T)}}=\left(\frac{d_{m}}{D}+\frac{1}{2}\right) \sum_{j=0}^{j=Z-1} Q_{j} f_{k j}
$$

where: $M_{1(T)}$ is the friction torque of the bearing, $Q_{j}-j$-th equivalent load at the contact site of the ball and raceway, $Z$ - number of balls in the bearing, $d_{m}$ - pitch diameter of the bearing, $D$ - diameter of the ball, $f_{k j}-j$-th rolling friction coefficient of the ball on the raceway.

For verification, a developed extended contact model has been assumed (Kosmol, 2016), in which

$$
Q_{j}=Q_{i}+Q_{o}
$$

where: $Q_{i}, Q_{o}$ are contact loads on the internal $i$ and external $o$ raceway. In the model, the rolling friction coefficients are the same for every ball in the bearing. The value of this coefficient has been assumed arbitrarily.

For such assumptions, the total friction torque of the bearing can be presented as follows

$$
M_{1_{(T)}}=Z\left(\frac{d_{m}}{D}+\frac{1}{2}\right)\left(Q_{i}+Q_{o}\right) f_{k}
$$

By converting relationship (4.3) and assuming $M_{1(T)}=M_{o p}$, we can write down

$$
Q_{i}+Q_{o}=\frac{M_{o p}}{2 Z\left(\frac{d_{m}}{D}+\frac{1}{2}\right) f_{k}}
$$

where $M_{o p}$ is the friction torque measured at the research site. 
In relationship (4.4), the fact has been considered that at the research site there are two angular bearings.

For the tested angular bearings FAG 7013B, relationship (4.4) assumes the following form

$$
Q_{i}+Q_{o}=\frac{M_{o p}}{309.7 f_{k}}[\mathrm{~N}]
$$

where $M_{o p}$ is in Nmm and $f_{k}$ in $\mathrm{mm}$.

Relationship (4.5) allows indirect verification of the developed extended contact model through comparing the sum of contact loads calculated analytically and determined experimentally for arbitrarily assumed rolling friction coefficients.

Figure 10 portrays a comparison of the sum of contact loads $Q_{i}+Q_{o}$ determined on the basis of experimental research as per relationship (4.5), with recourse to the extended model and with recourse to the classical model and the given FEM simulation results.
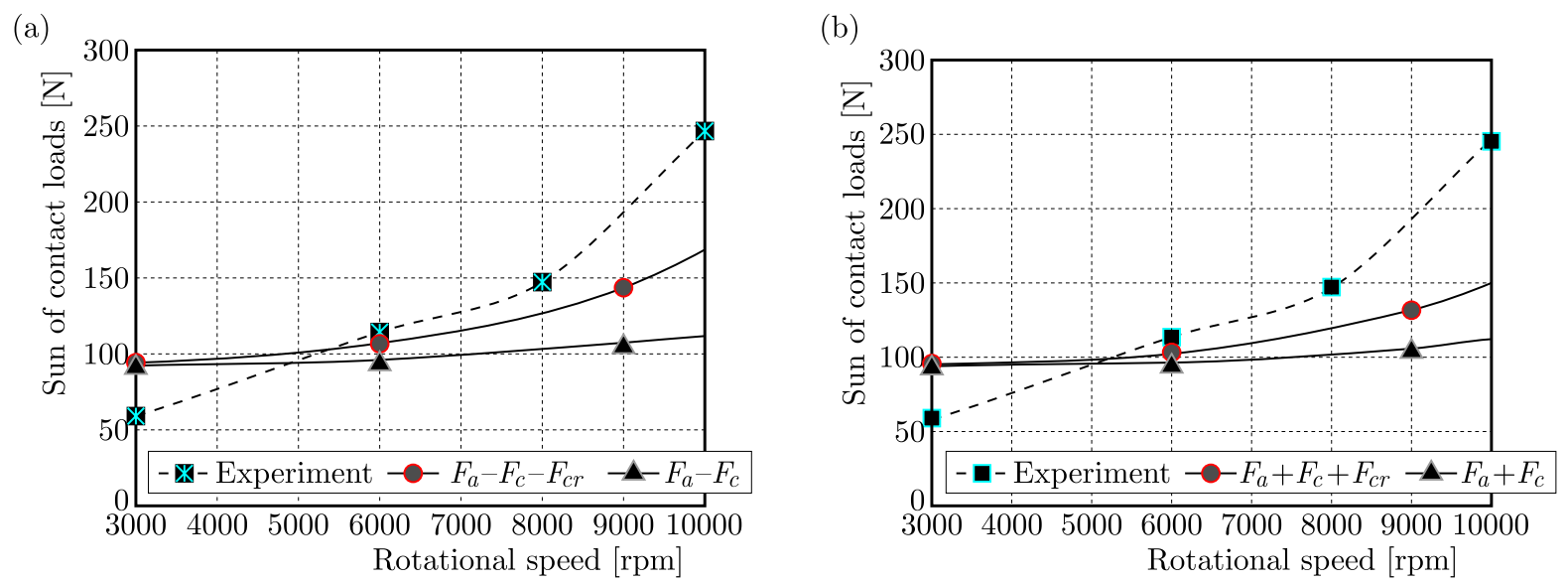

Fig. 10. Comparison of sums of contact loads $Q_{i}+Q_{o}$ obtained from experimental research and (a) analytical tests, (b) FEM simulation: $F_{a}+F_{c}+F_{c r}$ - extended model, $F_{a}+F_{c}$ - classical model, $f_{k}=0.0029-$ friction coefficient, $F_{a}=500 \mathrm{~N}$ - preload of the bearing

The comparison of the sums of contact loads obtained from the experimental research (relationship (4.5)) with the analytical tests and FEM obtained from the extended model (Fig. 10) demonstrates their reasonable consistence, but qualitatively only. Over $6000 \mathrm{rpm}$, an increase in the experimental sum of contact loads is much more similar to the results obtained for the extended model than for the classical model. This can be interpreted as an indirect confirmation of the correctness of the extended model. But below $6000 \mathrm{rpm}$, the experimental and theoretical results differ. It means that the motion resistance depends not only on the friction torque due to load (4.1) but on other resistances, for example on the spinning effect or cage resistance, too (Kosmol, 2016).

Figure 10 presents also the results in the form of the sum of contact loads for the classical model. They show that the sums of contact loads are explicitly smaller (by approx. $20 \%$ and more) from the results obtained from the extended model and from the experiment.

The results shown in Fig. 10 have been obtained by fine-tuning of the friction coefficient $f_{k}$ (see the relationship (4.5)) for the best match with the analytical results and FEM. The value of the friction coefficient fine-tuned in that way amounts to approx. 0.0029, i.e. it is in the range which has been presented in publications on angular rolling bearings, i.e. 0.002-0.005. 


\section{Summary}

The article presents a new contact model of an angular bearing, called the extended model. The model takes into account the effects of the centrifugal load caused by the rotating ring of the bearing and the issue of elasticity. Present models, encountered in literature and referred to in the article as classical, consider the centrifugal force caused by the rotating ball only. Results of analytical research on the extended model and FEM simulations show explicitly that the contact angles of bearings, and thus the contact loads, differ very much from those values obtained in the classical model. These differences are disadvantageous while designing bearing assemblies, since the contact loads are greater than those obtained in the classical model. This means, among others, that the present structures with angular contact bearings are in reality subject to greater loads than the constructor has envisaged.

The motto of the article is to design analytical calculation models in such a form which would enable the constructor to estimate the contact loads using just a standard scientific calculator or MS Excel type applications, so that there would be no need for solving complex models using numerical methods.

The article also features the results of verification of the developed extended model. The verification encompassed both, FEM numerical analysis and experimental investigation. The results of the verification (experimental research and FEM simulation) have confirmed relative consistence with the results of the analytical examination developed on the basis of the extended model. They have shown that the present classical model leads to an erroneous evaluation of contact loads in the bearing.

Hence, the article formulates a viewpoint that for engineering purposes, the suggested extended contact model enables calculation of contact loads in angular bearings with a sufficient practical accuracy.

\section{References}

1. Abele E., Altintas Y., Brecher C., 2010, Machine tool spindle units, CIRP Annals - Manufacturing Technology, 59, 781-802

2. Alfares M.A., ElsharkaWy A., 2003, Effects of axial preloading of angular contact ball bearings on the dynamics of a grinding machine spindle system, Journal of Materials Processing Technology, 136, $48-59$

3. Altintas Y., CaO Y., 2005, Virtual design and optimization on machine tool spindles, Annals of the CIRP, 54, 1, 379-382

4. Antoine F., Abba G., Molinari A., 2005, A new proposal for explicit angle calculation in angular contact ball bearing, Journal of Mechanical Design, 128, 2, 468-478

5. Cao Y., Altintas Y., 2007, Modelling of spindle-bearing and machine tool systems for virtual simulation of milling operations, International Journal of Machine Tools and Manufacture, 47, $1342-1350$

6. Chen J.-S., Hwang Y.-W., 2006, Centrifugal force induced dynamics of motorized high-speed spindle, International Journal of Machine Tools and Manufacture, 30, 10-19

7. Chojnacki M., 2016, Experimental research of motion resistance in angular bearings (in Polish), M.Sc. Thesis, Silesian University of Technology, Gliwice

8. Harris T., Kotzalas M., 2013, Essential Concepts of Bearing Technology. Rolling Bearing Analysis, 5th Ed., Taylor \& Francis Group, London

9. JęDrZEJEWski J., KwAŚnY W., 2010, Modelling of angular contact ball bearings and axial displacements for high-speed spindles, CIRP Annals-Manufacturing Technology, 59, 377-383 
10. JiAng S., MaO H., 2010, Investigation of variable optimum preload for machine tool spindle, International Journal of Machine Tools and Manufacture, 50, 19-28

11. Kosmol J., 2016, Determination of Motion Resistances in High-Speed Spindle Angular Bearings, Monograph, Silesian University of Technology Publisher, Gliwice

12. Kosmol J., Gatys R., 2016, Simulation research of the influence of rotational speed on contact loads in angular bearings (in Polish), Inżynieria Maszyn, 21, 1, 32-45

13. Musią J., Styp-Rekowski M., 1999, Analytical and experimental method of determination of the coefficient of motion resistance in rolling friction (in Polish), Proceedings of Conference "Problems of Unconventional Bearing Configuration", Łódź, 59-65

14. MuszyŃski M., 2017, Experimental research of motion resistance in angular bearings (in Polish), M.Sc. Thesis, Silesian University of Technology, Gliwice

15. Noel D., Rithou M., Furet B., Leloch S., 2013, Complete analytical expression of the stiffness matrix of angular contact ball bearing, Journal of Tribology, 135, 4

16. Palmgren A., 1951, Rolling Bearings (in Polish), PWT, Warszawa, 235

17. Styp-Rekowski M., 1999, Problems of the Internal Resistance of Motion in an Angle Bearing (in Polish), University Press of Technology and Agriculture Academy in Bydgoszcz, Thesis, 99, Bydgoszcz

Manuscript received May 16, 2017; accepted for print June 30, 2018 